by compensatory troughs during the postadolescence period. In other words, are the peaks due to the acceleration of a process that, without the intervention of puberty, would have proceeded at a more leisurely pace and become manifest as cancer somewhat later? Some of the graphs in Lee's paper ${ }^{1}$ seem to suggest that this might be the case. Recently Alice $M$. Stewart and G. W. Kneale ${ }^{4}$ reported from the department of social medicine in Oxford that the death rates for neuroblastomas, Wilms's tumours, and lymphatic leukaemias had peaks at different ages in the children of mothers exposed to $x$ rays for the purpose of pelvimetry during pregnancy. Do these peaks correspond to periods of maximum growth rates in the tissue concerned?

Studies both on laboratory animals and on man repeatedly point to cancer as having a multiplicity of causes. It might therefore be unwise to develop theories of cancer causation from studies of death rates among populations whose degree of exposure to carcinogenic hazards is unknown. The fact that there are peaks in the death rates from acute myeloid leukaemia and bone cancer shortly after puberty may be telling us no more about the true aetiology of these forms of malignant disease than that a period of rapid growth, or a period of hormonal change or instability, temporarily upsets the body's natural defences against the proliferation of cancerous cells. But for puberty, cells that had been rendered cancerous because of previous exposure to a carcinogen might have been held in check for longer or for ever-perhaps even destroyed.

\section{Anaesthesia and Drugs}

Many problems are associated with the interaction of drugs. Examples of potentiation, synergism, and inhibition are regularly reviewed in the literature, and doctors are becoming increasingly aware of some of the hazards of drug combinations. ${ }^{1}$

A recent report ${ }^{2}$ illustrates an unusual interaction involving anaesthesia and diphenylhydantoin-a commonly used anticonvulsant for epilepsy. A child already showing nystagmusa sign of early toxicity from hydantoin-was given a general anaesthetic which incorporated halothane for cystoscopy of $1 \frac{1}{2}$ hours' duration. Initial recovery from anaesthesia was unduly prolonged, and the patient became pyrexial. There was then a further temporary lapse into coma. At this time the levels of both diphenylhydantoin and serum aspartate aminotransferase (S.G.O.T.) in the blood were unduly high. Only when diphenylhydantoin was withdrawn for 48 hours did the child make an uninterrupted recovery, with a return to normal levels of S.G.O.T. and diphenylhydantoin.

Diphenylhydantoin is detoxified in the liver and depends on parahydroxylation of one phenyl group. ${ }^{3}$ Some patients have a familial inability to carry it out. In other patients certain drugs (chlorpromazine, perchlorperazine, chlordiazepoxide, oestrogens, anticoagulants ${ }^{4}$ ) have also been blamed for interfering with the detoxification process. Patients suffering

Prescott, L. F., Lancet, 1969, 2, 1239.

2 Karlin, J. M., and Kutt, H., Fournal of Pediatrics, 1970, 76, 941

3 Kutt, H., Winters, W., Kokenge, R., and McDowall, F., Archives of Neurology, 1964, 11, 642 .

- Kutt, H., and McDowell, F., fournal of the American Medical Association, 1968, 203, 969.

5 Hansen, J. M., Kristensen, M., Skovsted, L., and Christensen, L. K., Lancet, 1966, 2, 265.

- Stephen, C. R., et al., Anesthesiology, 1958, 19, 197.

- Mushin, W. W., Rosen, M., Bowen, D. J., and Campbell, H., British Medical fournal, 1964, 2, 329. from liver disease may also have a low tolerance to diphenylhydantoin.

It is well attested ${ }^{6}$ that general anaesthesia causes a temporary interference with liver function, though it may be difficult to differentiate between the effect of the anaesthetic itself and the effects of such things as hypoxia, hypercarbia, hypotension, or malnutrition, to say nothing of the question of dosage, duration, or repetitive nature of the administration. In this respect halothane is no different from any other anaesthetic. " But it seems at least probable that in the case referred to the aggravation of the signs of diphenylhydantoin poisoning was a result of the anaesthetic on hepatic function.

The mechanisms of biotransformation for a wide range of drugs are still imperfectly understood. Consequently there is no way of confidently forecasting the effects on them of the general cellular depression produced by anaesthesia. But it is clearly important for anaesthetists invariably to ask their patients - as indeed they normally do-about any drugs they may be taking, including self-medication. It is particularly with drugs taken on a long-term routine basis, such as tranquillizers, hypnotics, and anticonvulsants, and about which anaesthetists may not be fully informed, that troubles may arise.

\section{Super Rats?}

Rats multiplied in many towns as a result of last year's dustmen's strikes. The prospect of another wave of disputes, with vast accumulations of refuse, will therefore give rise to concern. When the anticoagulant rodenticide warfarin was first introduced in 1952 it was found to be so effective in destroying rats that heavy infestations in places such as sewers, warehouses, and dwellings could be eradicated relatively easily by its proper use. Moreover, once cleared of rats an area could be kept virtually free of them by keeping anticoagulant baits permanently available.

Unfortunately, from 1958 onwards pockets of warfarinresistant rats began to be encountered in Britain and elsewhere. This resistance is a genetically transmitted trait, and hence populations of anticoagulant-resistant rats have become established in certain parts of the country. Other anticoagulant substances have been tested by the Ministry of Agriculture but so far without success. Chemosterilants, which could lead to the extinction of the rat population, have not yet found an application in this field. So the search for new and effective rodenticides must continue.

In the meantime we must fall back on using zinc phosphide in those areas where warfarin resistance is known to exist. In capable hands zinc phosphide can give good results, but it must be used skilfully because rats quickly become bait-shy when acute poisons are used. It is estimated that at present only a small area of the entire country-less than $2 \%$-has warfarin-resistant rat populations, and therefore this anticoagulant rodenticide can still be used extensively. Unfortunately, many local authorities do not take rodent control as seriously as they should and are reluctant to spend money on an effective rodent control service. While it may not be possible at present to produce a town free of rats, much more could be done to control rodent pests, in both urban and rural areas.

Rats and mice as vectors of disease may have little medical significance in Britain. Nevertheless, the considerable damage they do to food and property is of appreciable importance to the national economy. 